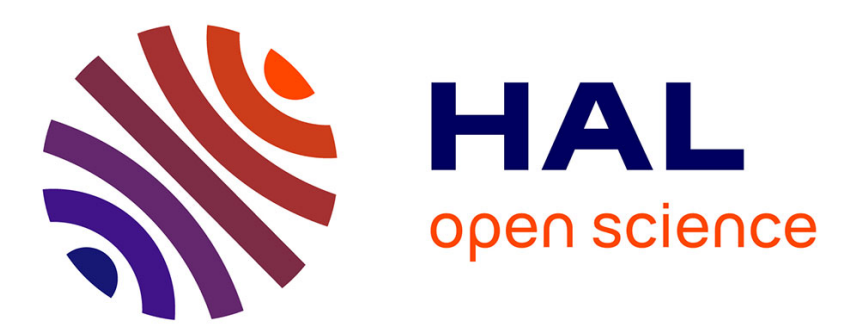

\title{
Characterization, modeling and robust control of a nonlinear 2-dof piezocantilever for micromanipulation / microassembly.
}

Micky Rakotondrabe, Joël Agnus, Kanty Rabenorosoa, Nicolas Chaillet

\section{To cite this version:}

Micky Rakotondrabe, Joël Agnus, Kanty Rabenorosoa, Nicolas Chaillet. Characterization, modeling and robust control of a nonlinear 2-dof piezocantilever for micromanipulation / microassembly.. IEEE/RSJ International Conference on Intelligent RObots and Systems, IROS'09., Oct 2009, SaintLouis, Missouri, United States. pp.767-774. hal-00432526

\section{HAL Id: hal-00432526 \\ https://hal.science/hal-00432526}

Submitted on 16 Nov 2009

HAL is a multi-disciplinary open access archive for the deposit and dissemination of scientific research documents, whether they are published or not. The documents may come from teaching and research institutions in France or abroad, or from public or private research centers.
L'archive ouverte pluridisciplinaire HAL, est destinée au dépôt et à la diffusion de documents scientifiques de niveau recherche, publiés ou non, émanant des établissements d'enseignement et de recherche français ou étrangers, des laboratoires publics ou privés. 


\section{Characterization, modeling and robust control of a nonlinear 2-dof piezocantilever for micromanipulation/microassembly}

Micky Rakotondrabe, Member, IEEE, Joël Agnus, Kanty Rabenorosoa and Nicolas Chaillet, Member, IEEE

\begin{abstract}
Piezoelectric cantilevers have proved their performances for actuating microgrippers dedicated to micromanipulation and microassembly tasks. While the control of one degree-of-freedom (dof) piezocantilevers have been well whelmed, the control of multi-dof one has not been addressed. Indeed, to assemble complex structures, the use of multidof microgrippers is recognized. Unfortunately, strong coupling between the axis and nonlinearities indeniably limit their performances.

This paper deals with the modeling and control of a piezocantilever that has 2 degrees of freedom: in-plane and out of plane deflections. While such a characteristic allows the microgrippers perform both orientation and translation during micromanipulation/microassembly tasks, the strong coupling between the two dof makes difficult their control. Moreover, nonlinearities (hysteresis and creep) raise when the piezocantilever is used in high deformation. To overcome these, we consider the coupling as a disturbance, model the nonlinearities with the quadrilateral approximation and we apply a robust $H_{\infty}$ controller that accounts them. The experiments show the efficiency of the synthesized controller and the obtained performances are convenient for micromanipulation/microassembly tasks.
\end{abstract}

\section{INTRODUCTION}

Face to the need of small products that have several functions (sensing, actuation, ...) and natures (mechanical, electrical, optical, ...), micromanipulation/microassembly tasks become more and more studied and used in laboratories nowadays. To perform such tasks, systems with high performances should indeniably be utilized. In most of cases, these systems are based on active materials because hinges based elements derive frictions and then imprecisions. Piezoelectric materials are among the most utilized materials. While they offer both high resolution and low response time, they can be used for sensing and for actuation. One of their actuation applications is the piezoelectric microgripper [1][2][3][4].

A piezogripper (piezoelectric microgripper) is composed of two piezoecantilevers (piezoelectric cantilevers) working in deflection (Fig. 1). The first piezocantilever is used to position the manipulated micro-object whilst

FEMTO-st Institute,

UMR CNRS-6174 / UFC / ENSMM / UTBM

Automatic Control and Micro-Mechatronic Systems department (AS2M department)

25000 Besançon - France

corresponding author: mrakoton@femto-st.fr the second one is used to control the force, both along the $y$-axis [5]. On the one hand, despite the difficulty to measure or estimate the force, the force control in a piezocatilever is being emerging [6]. On the other hand, the deflection control of a piezocantilever is nowadays overcome, [7] gives a survey of several applied techniques.

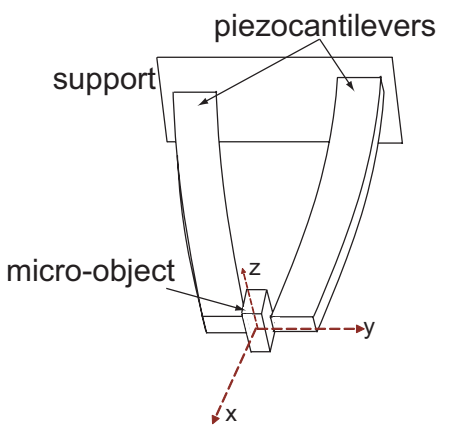

Fig. 1. Scheme of a piezoelectric microgripper made up of two piezocantilevers.

If the development and control of 1-dof piezocantilevers based piezogrippers are enough advanced, the tasks that they can performed are limited to either rotation or translation but not the combination of both. Hence, Agnus et al. developed a two-fingered piezoelectric microgripper (Fig. 2)[8], each finger being able to move independently from the other in two orthogonal directions: in plane and out of plane deflections. A finger is made up of a 2-dof piezocantilever and eventually a tool. Here, in plane deflection is defined as the deflection in the same plane than a piezocantilever electrodes. This microgripper has therefore four articular dof able to grip, hold and release submillimetric-sized objects in the $y$-axis. The fingers can also move up and down $(z$ axis) for fine motions like micro-insertion tasks. Finally, they are able to orientate (around $x$-axis) micro-objects by combining opposite out-of-plane motions of the fingers. The microgripper characteristics are very useful in micromanipulation/microassembly and have proved its utilities in micromanipulation of watch screws or in microspectrometers alignment tasks [10]. 


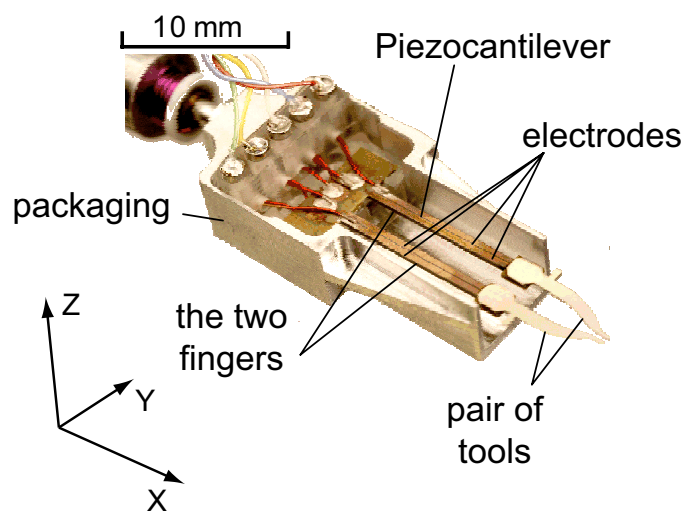

Fig. 2. Picture of the microgripper.

Notwithstanding, when applying a reference deflection along $y$-axis (resp. $z$-axis), a residual deflection is obtained in the $z$-axis (resp. $y$-axis). This is due to mechanical imperfection of the structure and particularly to the misalignment of the electrodes. This coupling makes very inaccurate the system because unwanted movements appear during micromanipulation/microassembly tasks. In addition, hysteresis and creep phenomena become non-negligible when the applied electrical field is large. The applied voltage should effectively be relatively enough in order to cover the required deflection ranges. This paper presents the deflection control of the 2-dof piezocantilever in taking into account the axis coupling and the nonlinearities. A robust $H_{\infty}$ controller is used because model uncertainty due to the hysteresis must be taken into account and specifications relative to performances required for tasks should be ensured. The paper is organized as follows. First, the 2-dof piezocantilever is presented. After that, we present its characteristics, notably the hysteresis and coupling parts. Then, the modeling and robust control follow the section. Finally, experimental results end the paper.

\section{Presentation of the 2-Dof Piezocantilever}

The functioning principle of one finger of the microgripper is based on a piezoelectric cantilever with local electrodes, called 'duo-bimorph', for which a static modelling has been established [9]. It can be assimilated to a cantilever beam clamped at one end. The two dof are obtained with a judicious application of voltages on the piezoelectric plates.

Fig. 3 pictures the functioning of the actuator with cross section views. The structure at rest is presented with solid area and the deformed actuator with dashed lines. $\mathbf{P}$ indicates the polarisation of the piezoelectric material (Fig. 3-a). In the first configuration (Fig. 3-b), the four electrodes are set to the same voltage $V_{Z}>0$. The upper layer stretches along $x$ while the lower layer contracts, which leads to a flexion along $-z$, as the layers are glued

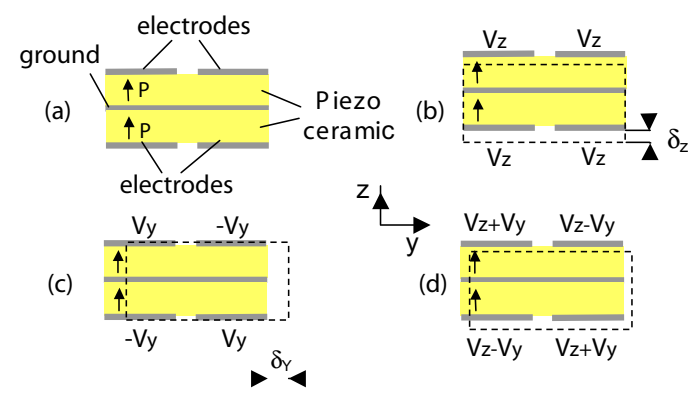

Fig. 3. Cross section of a gripper finger (Y-Z plane): working principle of the actuator.

one to each other. In the second situation (Fig. 3-c), the voltages on two adjacent and two opposite electrodes are $V_{Y}$ and $-V_{Y}\left(V_{Y}>0\right)$; the electric fields are in the same direction as the polarisation in the left half-part of the beam and in the opposite direction in the right half-part of the same beam. Thus, the left part stretches while the right part contracts, which leads to a deflexion along $y$. Finally, in the last configuration (Fig. 3-d), the electrodes are set at voltages $V_{Z}+V_{Y}$ and $V_{Z}-V_{Y}$, which yields a deflexion in $y$ and $z$ directions. The four articular dof (two per finger) of the gripper are useful to perform gripping, insertion or rotation tasks. They also permit to correct a possible misalignment of the tools. In this paper, we are interested by the modeling and control of one finger (piezocantilever).

\section{OPEN-LOOP ANALYSIS}

In this section, we characterize the piezocantilever. The experimental setup is based on:

- one 2-dof piezocantilever,

- two optical sensors to measure the deflections of the two axis (Fig. 4-a). Each sensor (Keyence-2420) has $10 \mathrm{~nm}$ of resolution,

- a computer-DSpace hardware and the MatlabSimulink (c) software for the data-acquisition and control,

- and a voltage amplifier.
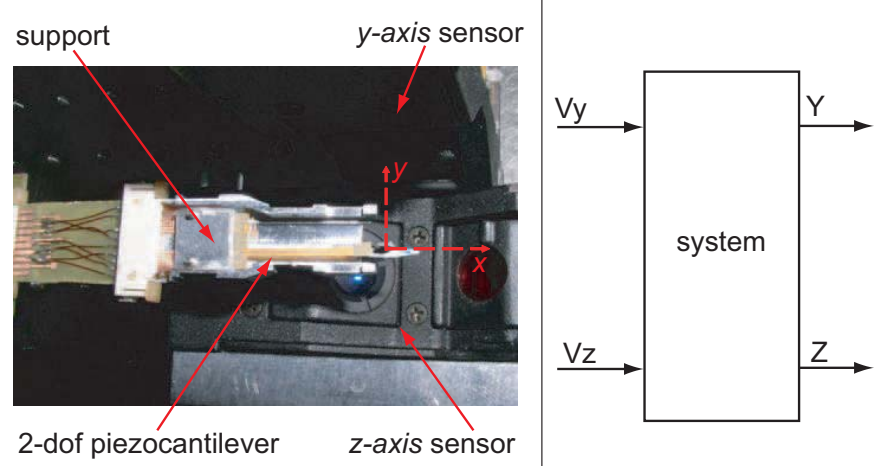

(a)

Fig. 4. The setup. 
The piezocantilever can be considered as a MIMO system with two-inputs and two ouptuts (Fig. 4-b). In the sequel we analyze the nonlinearities (hysteresis and creep) and the vibration of the system. It has been shown that for piezocantilevers, the hysteresis only affect the static part while the creep can be considered as an additional disturbance [11]. Moreover the hysteresis, the vibration during the transient part and the creep can be separately analyzed and identified. The vibration and the creep will be characterized using a step input. The former is the transient part of the response while the latter is the drift that appears after the end of the transient part. The hysteresis will be characterized using a sine input voltage. The frequency of the sine input is chosen such as it is not too low (to avoid the creep effect) and not too high (to avoid the dynamic part effect).

\section{A. Dynamic analysis and vibration compensation}

First, we analyze the transient part of the system. The analysis is performed as follows. A step voltage $V_{y}=20 \mathrm{~V}$ is first input while $V_{z}=0$. The transient part of the obtained deflection $Y$ is plotted. At the same time, its effect on the deflection $Z$ is also plotted. After that, a step voltage $V_{z}=20 \mathrm{~V}$ is applied and $V_{y}$ is reset to zero. Thus, the direct deflection $Z$ and the resulting coupling $Y$ are plotted. The four results are pictured in the Fig. 5 .

Fig. 5-a and Fig. 5-d respectively indicate that the transient parts of the direct transfers $V_{y} \rightarrow Y$ and $V_{z} \rightarrow Z$ are very oscillating. In micromanipulation/microassembly tasks, vibrations are unwanted. In fact, a microgripper with oscillating piezocantilevers may destroy a breakable manipulated micro-object if the overshots are high. Elsewhere, the coupling effect $V_{z} \rightarrow Y$ is also oscillating (Fig. 5-b). Despite that, the coupling effect $V_{y} \rightarrow Z$ is low (less than $3 \mu m$ ) (Fig. 5-c).
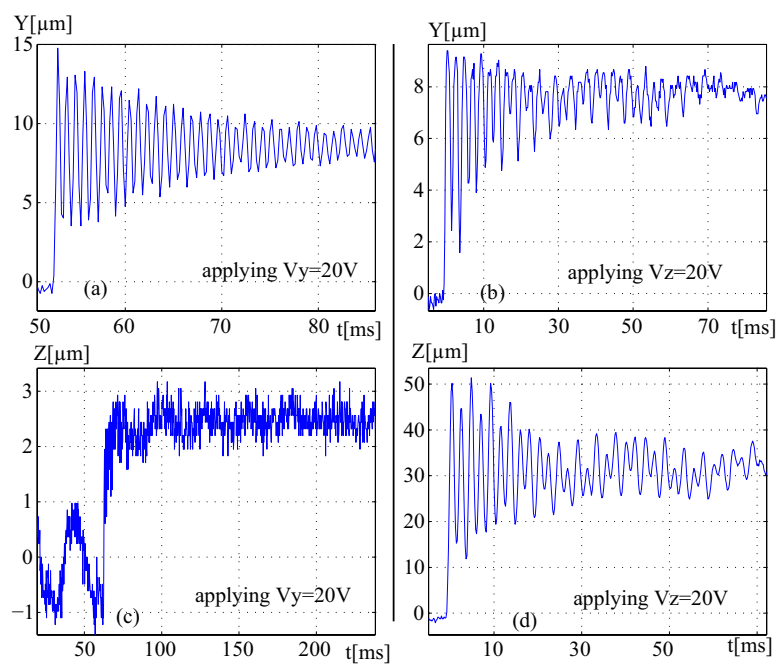

Fig. 5. Transient part characteristics: experimental results.
In closed-loop technique, it is very difficult to synthesize controllers for oscillating systems if the specified settlingtime is largely low relative to the settling-time of the vibration. So, before synthesizing a closed-loop controller, we propose to attenuate the vibration. We choose the input-shaping Zero-Vibration technique [12] because of its ease of implementation. Because of the limited length of the paper, this part will not be detailed in this paper. However, the readers can see more detail on its computation and application for one dof piezocantilever in our previous work [14]. In this paper, to compensate the vibration, we do not consider the coupling between the axis:

- one compensator is used to compensate the vibration of the transfer $V_{y} \rightarrow Y$. The compensator computation is based on the step response pictured in Fig. 5-a,

- another compensator is used for the transfer $V_{z} \rightarrow$ $Z$. It is computed using the Fig. 5 -d.

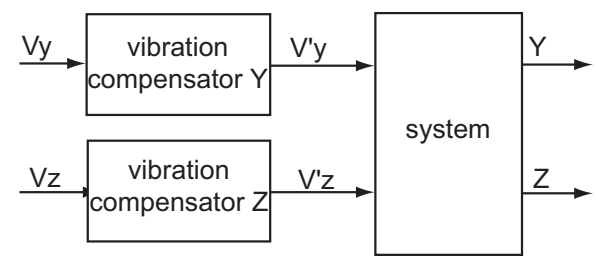

Fig. 6. Scheme of the piezocantilever with vibrations compensation.

Fig. 6 presents the bloc-scheme of the piezocantilever with the vibration compensators. In the figure, $V_{y}$ and $V_{z}$ are the input voltages while $V_{y}^{\prime}$ and $V_{z}^{\prime}$ are the new inputs of the piezocantilever. The experimental results are interesting and are pictured in the Fig. 7. When a step input $V_{y}=20 \mathrm{~V}$ is applied, the obtained deflection $Y$ is henceforth without vibration (Fig. 7-a)). Similarly, when a step input $V_{z}=20 \mathrm{~V}$ is applied the initial vibration of the deflection $Z$ is attenuated (Fig. 7-d). Finally, the vibration of the coupling transfer has been attenuated (see Fig. 7-b and -c respectively).

\section{B. Static analysis}

Here, we analyze the static characteristics, i.e. the behaviors in the voltages-deflections plane, of the piezocantilever. For that, we use sine input voltages. For the experimented piezocantilever, various experiments lead us to choose a frequency of $0.5 \mathrm{~Hz}$. The results are pictured in Fig. 8.

Fig. 8-a shows the deflection $Y$ vs a sine applied voltage $V_{y}$ and for three constant values of $V_{z}$. We first remark that the direct transfer $V_{y} \rightarrow Y$ is hysteretic. Secondly, it appears that this transfer is influenced by the constant input $V_{z}$. Indeed, not only the hysteresis 

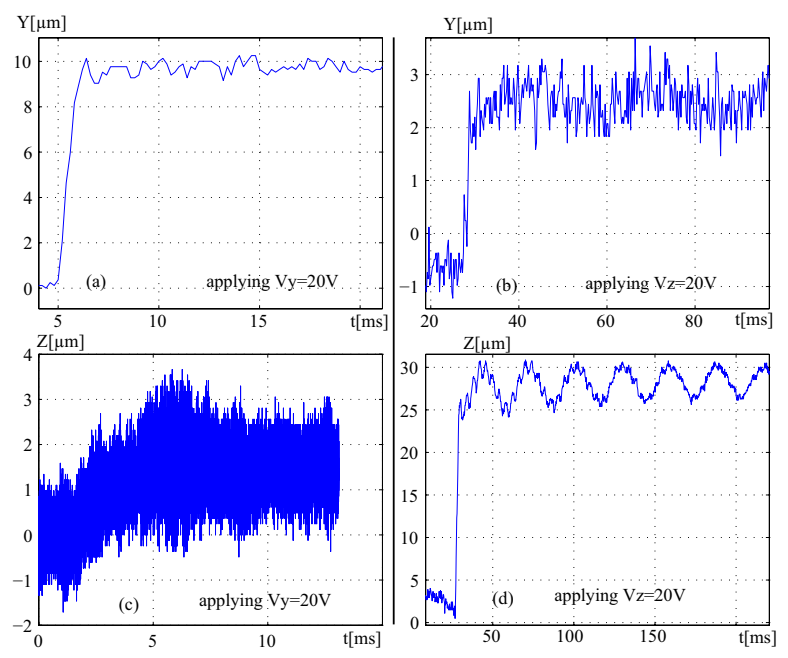

Fig. 7. Transient part characteristics when using vibrations compensation: experimental results.

obtained with $V_{z}=20 \mathrm{~V}$ is slightly angled relative to others but the three curves are shifted each other. To well understand the coupling and more evaluate the shift, we apply a sine voltage $V_{z}$ and plot the resulting deflection $Y$ (Fig. 8-b). It appears that the coupling is nonlinear and the effect of $V z$ on $Y$ ranges between $\pm 5 \mu m$ for $V_{y}=0$.

Similarly to the previous experiments, Fig. 8-d shows the deflection $Z$ vs a sine applied voltage $V_{z}$ and for three constant values of $V_{y}$. Like $V_{y} \rightarrow Y$, we also remark that the direct transfer $V_{z} \rightarrow Z$ is hysteretic. However, it seems that whatever an applied constant $V_{y}$ is, the hysteresis curves are neither shifted nor angled themselves: the coupling seems negligible. To more analyze that, we apply a sine voltage $V_{y}$ and check its influence on the deflection $Z$. The results are plotted in the Fig. 8-c and clearly indicate that the coupling is negligible. Indeed, from the curves, we a priori have a low value of $\frac{\partial Z}{\partial V_{y}}$.

\section{Creep analysis}

Now let us analyze the creep phenomenon that may behave the piezocantilever. The creep is defined as the slow drift that appears after the transient part when a step voltage is applied. The amplitude of the creep depends on the amplitude of the input. In general, the transient part of piezocantilevers has a settling-time less than $100 \mathrm{~ms}$ whilst the creep has more than $3 \mathrm{~min}$.

The experiments are performed with a step $V_{y}=20 \mathrm{~V}$ while $V_{z}=0 \mathrm{~V}$ and then $V_{y}=0 \mathrm{~V}$ while $V_{z}=20 \mathrm{~V}$. To well observe the drift, the duration of the acquisition data is $600 \mathrm{~s}$. The results lead to the following remarks.

- Fig. 9-a: the creep in the transfer $V_{y} \rightarrow Y$ is considerable: more than $2.5 \mu m$ (i.e. $27 \%=\frac{2.5}{9}$, where $9 \mu \mathrm{m}$ is the final value before creep).

- Fig. 9-d: despite the noise, the creep in the transfer $V_{z} \rightarrow Z$ is also considerable: more than $10 \mu m$ (i.e. $\left.40 \%=\frac{10}{25}\right)$.
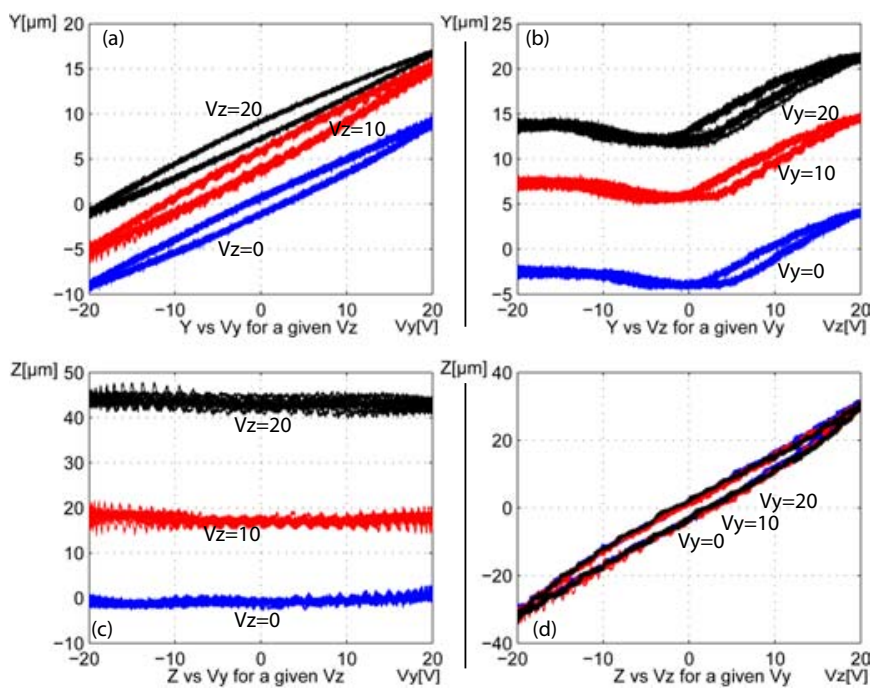

Fig. 8. Static characteristics: experimental results.

- Fig. 9-c: the effect of $V_{y}$ to $Z$ seems not providing any creep.

- Fig. 9-b: finally, the coupling effect of $V_{y}$ on $Z$ added with the creep is more than $3.5 \mu \mathrm{m}$. The whole should be rejected when closed-loop controling the piezocantilever.
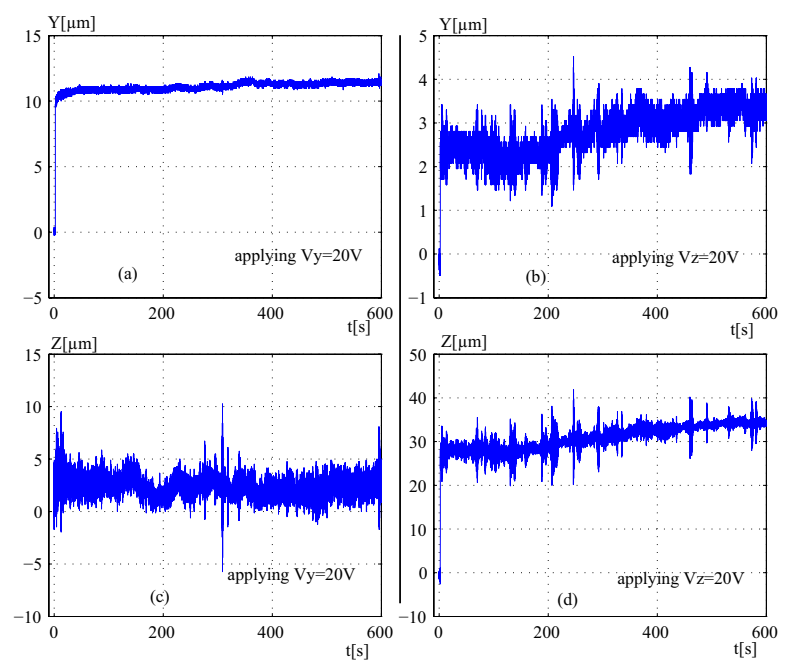

Fig. 9. Creep characteristics: experimental results.

\section{Modeling}

In order to synthesize a controller, we give a model of the system in this section. The considered system is now the 2-dof piezocantilever including the vibration compensators as shown in Fig. 6.

Consider the following multivariable nonlinear model:

$$
\left(\begin{array}{c}
Y \\
Z
\end{array}\right)=\Gamma\left(V_{y}, V_{z}, s\right) \Leftrightarrow\left\{\begin{array}{l}
Y=\Gamma_{y}\left(V_{y}, V_{z}, s\right) \\
Z=\Gamma_{z}\left(V_{y}, V_{z}, s\right)
\end{array}\right.
$$


where $\Gamma_{i}\left(V_{y}, V_{z}, s\right)$ represents a nonlinear operator dependent at the same time on the input $V_{y}$ and $V_{z}$. The reliance of $\Gamma_{i}$ on the Laplace variable $s$ means that it also includes dynamic parts. According to [11], nonlinear 1dof piezocantilevers can be described by a static hysteresis followed by a linear dynamic part. Moreover, the creep can be considered as an additional disturbance. This is expressed as follow:

$$
\delta=\Gamma(V, s)=\mathrm{H}(V) \cdot D(s)+C(V, s)
$$

where $\delta$ is the output, $\mathrm{H}(\mathrm{V})$ is the static hysteresis part that depends on the amplitude of $V, D(s)$ is the linear dynamic part and $C(V, s)$ is the creep part that depends on the amplitude of $V$ and has its own dynamic part. In some applications, the dynamic part inside $C(V, s)$ is approximated by a linear transfer function [15]. The result given by (equ 2) makes easy the analysis because we can first model the static part.

According to the Fig. 8-a, the hysteresis curves of $V_{y} \rightarrow Y$ are similar but only angled and shifted themselves when using different values of $V_{z}$. Because of the importance of the shift and the weakness of the tilt of the curves, we neglect the latter. However it will be considered during the controller synthesis. From these remarks, we can write:

$$
Y=\mathrm{H}_{y}\left(V_{y}\right)+\mathrm{T}_{V z \rightarrow Y}\left(V_{z}\right)
$$

where $\mathrm{H}_{y}\left(V_{y}\right)$ is the static hysteresis of $\left(V_{y} \rightarrow Y\right)$ at $V_{z}=0$ and $\mathrm{T}_{V z \rightarrow y}\left(V_{z}\right)$ represents a nonlinear operator modeling the coupling $\left(V_{z} \rightarrow Y\right)$. The latter can be expressed using one of the curves of Fig. 8-b.

Based on Fig. 8-c and Fig. 8-d, the similar remarks can be applied to the deflection $Z$. We have:

$$
Z=\mathrm{H}_{z}\left(V_{z}\right)+\mathrm{T}_{V y \rightarrow Z}\left(V_{y}\right)
$$

Fig. 8-c shows that $\mathrm{T}_{V y \rightarrow Z}\left(V_{y}\right)$ may be approximated by a linear function with low value of $\frac{\partial Z}{\partial V_{y}}$. However, in order to maintain the generality, we keep the notation $\mathrm{T}_{V y \rightarrow Z}\left(V_{y}\right)$.

According to the four figures of Fig. 7, each transfer has its own transient part.Based on (equ 2), we can write:

$$
\left\{\begin{array}{l}
Y=\mathrm{H}_{y}\left(V_{y}\right) \cdot D_{y}(s)+\mathrm{T}_{V z \rightarrow Y}\left(V_{z}, s\right)+C_{V y \rightarrow Y}\left(V_{y}, s\right) \\
+C_{V z \rightarrow Y}\left(V_{z}, s\right) \\
Z=\mathrm{H}_{z}\left(V_{z}\right) \cdot D_{z}(s)+\mathrm{T}_{V y \rightarrow Z}\left(V_{y}, s\right)+C_{V y \rightarrow Z}\left(V_{y}, s\right) \\
+C_{V z \rightarrow Z}\left(V_{z}, s\right)
\end{array}\right.
$$

where:

- $D_{y}(s)$ and $D_{z}(s)$ are respectively the dynamic parts of the direct transfers and can be identified using Fig. 7-a and Fig. 7-d,

- $\mathrm{T}_{V z \rightarrow Y}\left(V_{z}, s\right)$ and $\mathrm{T}_{V y \rightarrow Z}\left(V_{y}, s\right)$ are respectively the coupling transfers of $V_{z} \rightarrow Y$ and $V_{y} \rightarrow Z$. Each of these transfers include the static linear or nonlinear part (pictured in Fig. 8-b and Fig. 8-c) and the dynamic part (pictured in Fig. 7-b and Fig. 7-c),
- $C_{V y \rightarrow Y}\left(V_{y}, s\right)$ and $C_{V z \rightarrow Y} \cdot\left(V_{z}, s\right)$ are the creeps that appear in $Y$ when $V_{y}$ and $V_{z}$ are respectively applied,

- $C_{V y \rightarrow Z}\left(V_{y}, s\right)$ and $\left.C_{V z \rightarrow Z} V_{z}, s\right)$ are the creeps that appear in $Z$ when $V_{y}$ and $V_{z}$ are respectively applied.

During a micromanipulation/microassembly task, a piezogripper applies a force to the manipulated microobject. This manipulation force disturbs the functioning of each piezocantilever and may decreases its performances. So it is careful to account this force during the modeling and controller synthesis of the piezocantilever. As the force can be considered as an external disturbance, it can be easily introduced into (equ 5). We obtain:

$$
\left\{\begin{array}{l}
Y=\mathrm{H}_{y}\left(V_{y}\right) \cdot D_{y}(s)+\mathrm{T}_{V z \rightarrow Y}\left(V_{z}, s\right)+C_{V y \rightarrow Y}\left(V_{y}, s\right) \\
+C_{V z \rightarrow Y}\left(V_{z}, p\right)+s_{F_{y}} \cdot F_{y} \\
Z=\mathrm{H}_{z}\left(V_{z}\right) \cdot D_{z}(s)+\mathrm{T}_{V y \rightarrow Z}\left(V_{y}, s\right)+C_{V y \rightarrow Z}\left(V_{y}, s\right) \\
+C_{V z \rightarrow Z}\left(V_{z}, s\right)+s_{F_{z}} \cdot F_{z}
\end{array}\right.
$$

where $F_{y}$ and $F_{z}$ are the components of the external manipulation force respectively along $y$-axis and $z$-axis. The coefficients $s_{F_{y}}$ and $s_{F_{z}}$ are the compliance of the piezocantilever.

Assembling the coupling, the creep and the force into one disturbance, (equ 6) becomes:

$$
\left\{\begin{array}{l}
Y=\mathrm{H}_{y}\left(V_{y}\right) \cdot D_{y}(s)+d_{y} \\
Z=\mathrm{H}_{z}\left(V_{z}\right) \cdot D_{z}(s)+d_{z}
\end{array}\right.
$$

with $d_{y}$ and $d_{z}$ the disturbances respectively along $y$-axis and $z$-axis.

\section{A. Identification of the dynamic part}

The dynamic part $D_{i}(s)$ can be easily identified using the Fig. 7-a, Fig. 7-d and an ARMAX method. $D_{i}$ should be normalized, i.e. $D_{i}(0)=1$, because the gain is expressed by the static hysteresis. The identified models and the experimental results are presented in the Fig. 10a (for $V_{y} \rightarrow Y$ ) and Fig. 10-b (for $V_{z} \rightarrow Z$ ). Because the gains of the models are equal to one, the simulation pictured in the figures have been scaled in order to allow the comparison. We have:

$$
D_{y}(s)=\frac{(3333)^{2}}{(s+3333)^{2}}
$$

and

$D_{z}(s)=\frac{111\left(s+1.07 \times 10^{4}\right)\left(s^{2}-3.3 s+5.2 \times 10^{4}\right)}{\left(s^{2}+5.8 s+4.7 \times 10^{4}\right)\left(s^{2}+2023 s+1.4 \times 10^{6}\right)}$

\section{B. Approximation of the hysteresis}

In the literature, there are various models of hysteresis that have been applied to piezocantilevers, as exemple: the Prandtl-Ishlinskii model [14], the Preisach model [15], the Bouc-Wen model [16]. Despite the accuracy of these models, the synthesized controllers are often complex and 

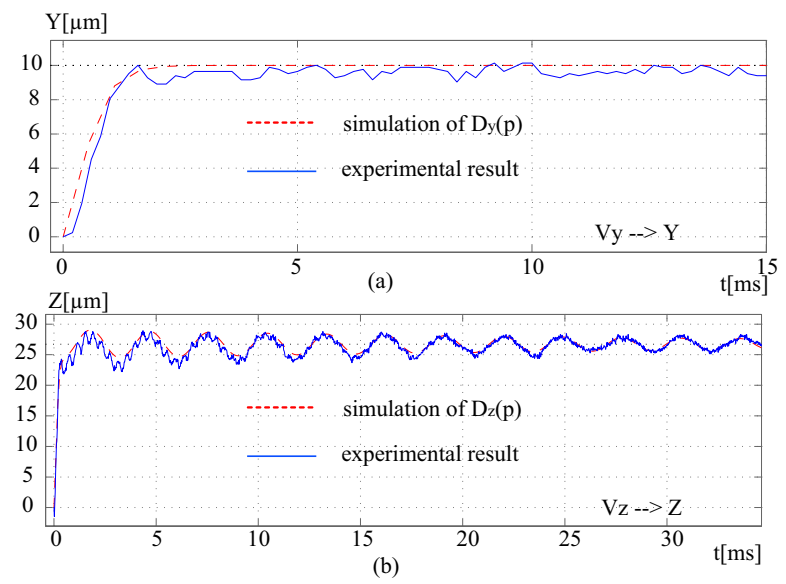

Fig. 10. Experimental result and simulation of the identified transient part.

nonlinear. Therefore, in order to use a linear controller, in our previous work [11], we proposed to approximate the 1dof static hysteresis by a linear expression with a nominal gain $\alpha$ subjected to a direct multiplicative input uncertainty $\Delta \cdot W_{e}$. In this approximation, the hysteresis curve is approximated by a quadrilateral (Fig. 11). The gain $\alpha$ is the slope of its axis. While $\Delta \in \mathbb{R}$ and $|\Delta| \leqslant$ 1 , the weighting $W_{e} \in \mathbb{R}$ is chosen to cover the radius $\frac{\alpha_{\max }-\alpha_{\min }}{2}$.

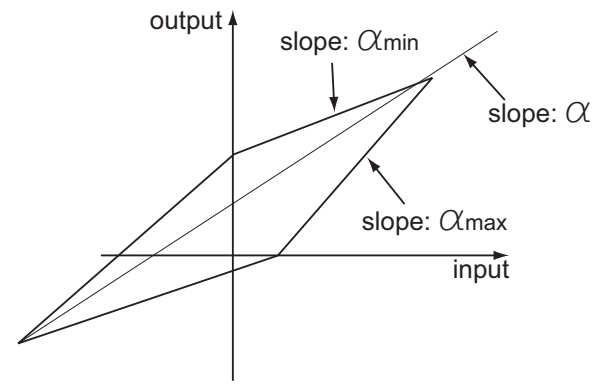

Fig. 11. Quadrilateral approximation of the hysteresis [11].

For $V_{z} \rightarrow Z$, the coefficients are easily computed using one of the three curves of Fig. 8-d. We have: $\alpha_{z}=$ $1.5\left[\frac{\mu m}{V}\right]$ and $W_{e z}=0.15\left[\frac{\mu m}{V}\right]$.

For $V_{y} \rightarrow Y$, we must take into account the tilt between the three hysteresis curves of Fig. 8-a. Hence, the maximal and minimal slopes, and then the weighting function $W_{e y}$, are computed using the three curves. However, $\alpha$ is only computed with one curve: we choose the hysteresis curve corresponding to $V_{z}=0 \mathrm{~V}$. We obtain: $\alpha_{y}=0.45\left[\frac{\mu m}{V}\right]$ and $W_{e z}=0.075\left[\frac{\mu m}{V}\right]$.

\section{Final model}

According to (equ 7), the initial coupled model becomes two independent models because the coupling parts were input inside the disturbance $d_{i}$. From the previous sub-section, each model is linear but subjected to an uncertainty. So, the controllers synthesis is based on the models presented in the Fig. 12. In the figure, $i$ denotes for $y$ or for $z$ and $\delta$ represents the deflection $Y$ or $Z$. $W_{e i}$ is the previous weighting function.

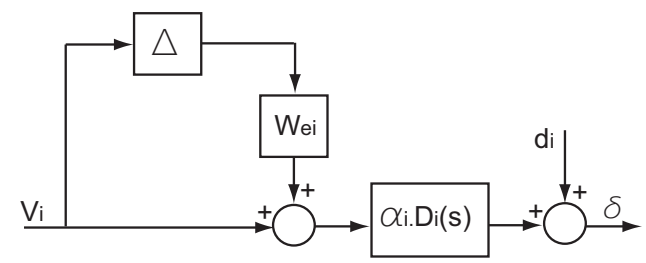

Fig. 12. Scheme of the models.

\section{V. $H_{\infty}$ CONTROL}

In this section we present the synthesis of a $H_{\infty}$ controller for each axis. Such a controller has been chosen to ensure the stability and the required performances despite the uncertainty and the coupling effects.

\section{A. Scheme and problem formulation}

During the controllers synthesis, the following points are taken into account:

- the stability should be ensured despite the presence of uncertainty on the static gain $\alpha_{i}$,

- the disturbance $d_{i}$ should be rejected,

- and the performances required for micromanipulation/microassembly tasks must be ensured.

For each system, the Fig. 13-a shows the closed-loop system with the weighting functions. From it, the standard scheme can be formed (Fig. 13-b). In the figure: $\delta_{\text {ref }}$ is the reference input, $K_{i}(s)$ is the controller to be synthesized, $W_{1 i}$ is the weighting for the pereformances specifications, $W_{2 i}$ is the weighting for the disturbance rejection and $W_{e i}$ is the previous weighting for uncertainty.

Using the two bloc-schemes of Fig. 13 and applying the standard $H_{\infty}$ problem [17], the problem consists in finding the corrector $K_{i}$ and an optimal value of $\gamma$ such as:

$$
\begin{gathered}
\left|S_{i}\right|<\frac{\gamma_{i}}{\left|W_{1 i}\right|}, \quad\left|S_{i}\right|<\frac{\gamma_{i}}{\mid W_{1 i} W_{2 i} i}, \\
\left|S_{i}\right|<\frac{\gamma_{i}}{\left|W_{2 i} W_{3 i}\right|}, \quad\left|S_{i} G_{i} K_{i}\right|<\frac{\gamma_{i}}{\left|W_{2 i} W_{3 i}\right|}
\end{gathered}
$$

where $G_{i}=\alpha_{i} D_{i}(s)$ is the nominal system and $S_{i}=$ $\frac{1}{1+K_{i} G_{i}}$ is the sentitivity function.

\section{B. Weighting functions}

1) For the y-axis: the following specifications are used to compute the weighting functions.

- the settling time is less than $40 \mathrm{~ms}$,

- the accuracy is better than $1 \%$ and the overshot is nul, 


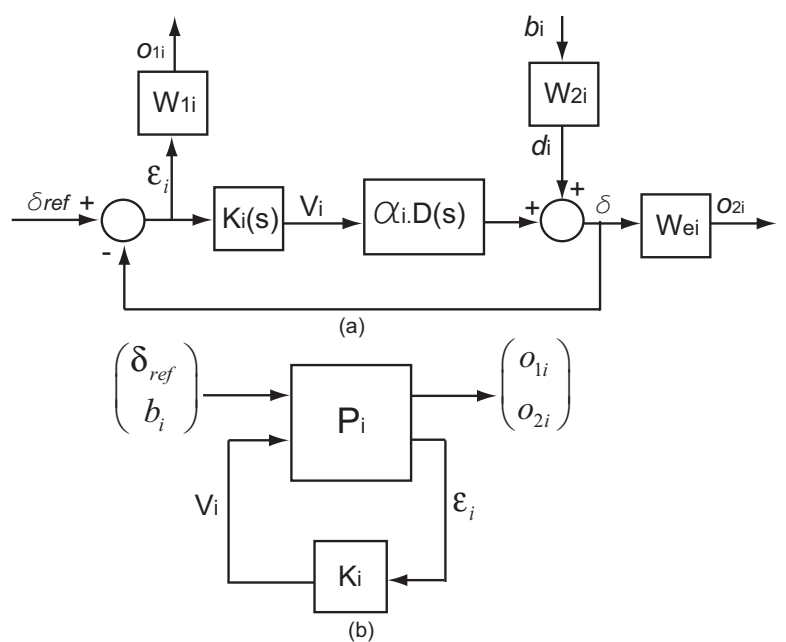

Fig. 13. (a): scheme of the closed-loop with weighting functions. (b) standard scheme.

- the settling time for the disturbance rejection is $30 \mathrm{~ms}$,

- when an external force $4 m \mathrm{~N}$, voltages of $V_{y}=20 \mathrm{~V}$ and $V_{z}=20 \mathrm{~V}$ are applied, they normally (i.e. in open-loop) generate an elastic deflection, a creep and a coupling of more than $15 \mu \mathrm{m}$. So we want that the maximal deflection in closed-loop due to them is less than $1 \mu m$.

From these, we choose:

$$
\left\{\begin{array}{l}
W_{1 y}=\frac{s+75}{s+0.75} \\
W_{2 y}=\frac{1.25(s+100)(s+0.75)}{(s+75)(s+7.5)}
\end{array}\right.
$$

2) For the z-axis: we use the following specifications.

- the settling time is less than $30 \mathrm{~ms}$,

- the accuracy is better than $1 \%$ and the overshot is nul,

- the settling time for the disturbance rejection is $20 \mathrm{~ms}$,

- when an external force $4 m N$, voltages of $V_{y}=20 \mathrm{~V}$ and $V_{z}=20 \mathrm{~V}$ are applied, they generate an elastic deflection, a creep and a coupling of more than $11 \mu \mathrm{m}$ in open-loop. So we want that the maximal deflection in closed-loop due to them is less than $1 \mu \mathrm{m}$.

From these, we choose:

$$
\left\{\begin{array}{l}
W_{1 z}=\frac{s+100}{s+1} \\
W_{2 z}=\frac{1.25(s+1)(s+0.18)}{(s+100)(s+0.013)}
\end{array}\right.
$$

\section{Computation of the controllers}

The controllers $K_{y}$ and $K_{z}$ have been computed using the Glover-Doyle algorithme [18][19]. Each of the corrector has respectively an order equal to 5 and 7 and have been reduced into 2 and 5 using the balanced realization technique [20]. We have:

$$
\left\{\begin{array}{l}
K_{y}=\frac{0.103(s+1683)(s+4.05)}{(s+7.5)(s+0.75)} \\
\gamma_{o p t}=1.6
\end{array}\right.
$$

and

$$
\left\{\begin{array}{l}
K_{z}=\frac{1301\left(s^{2}+5.8 s+4.7 \times 10^{4}\right)\left(s^{2}+2023 s+1.3 \times 10^{6}\right)}{(s+1)\left(s^{2}-11.7 s+8.7 \times 10^{4}\right)\left(s^{2}+7172 s+2 \times 10^{7}\right)} \\
\gamma_{o p t}=1.7
\end{array}\right.
$$

\section{EXPERIMENTAL RESULTS}

The two controllers have been implemented in a computer-Dspace material. The first experiments consist in applying a series of steps reference $Y_{\text {ref }}=10 \mu \mathrm{m}$ and $Z_{\text {ref }}=30 \mu \mathrm{m}$ and observing the coupling. The results show that the influence of an input $Z_{\text {ref }}$ on the $Y$-axis is rapidly rejected (zoom of Fig. 14-a) while the influence of $Y_{\text {ref }}$ on the $Z$-axis is negligible (zoom of Fig. 14-b).
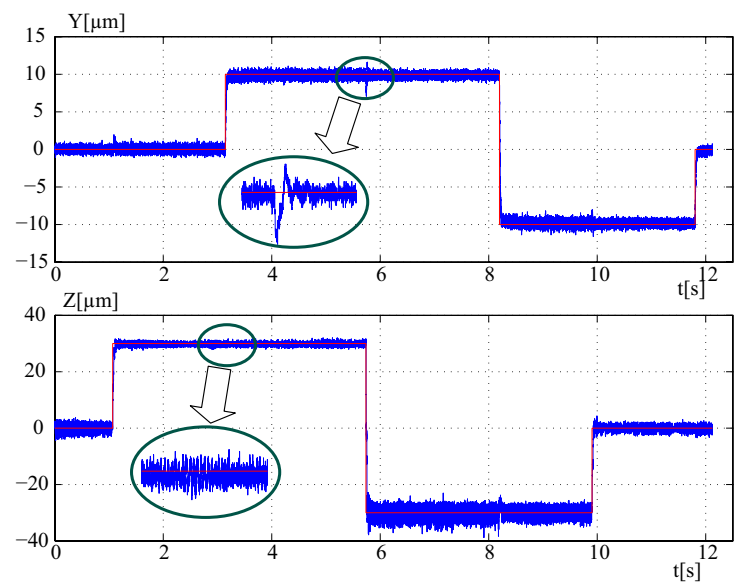

Fig. 14. Experimental coupling analysis of the closed-loop piezocantilever.

In the step responses, the settling time of $Y$ is lower than $40 \mathrm{~ms}$ and the overshot is null so that the wanted specifications are satisfied (Fig. 15-a). However, while the response of $Z$ presents a slight overshot, its settling time is nearly $60 \mathrm{~ms}$ (Fig. 15-b). The difference relative to the specifications is due to $\gamma=1.7$. Despite that, these results are well suited for micromanipulation/microassembly tasks.

Finally, a harmonic analysis is performed. For that, a sine input $Y_{\text {ref }}$ with $10 \mu \mathrm{m}$ of amplitude is applied. Fig. 16a pictures the responses $20 \log \left(\frac{Y}{Y_{\text {ref }}}\right)$ and $20 \log \left(\frac{Z}{Y_{\text {ref }}}\right)$. It shows that whatever the frequency is, the coupling is rejected $(Z$-magnitude less than $-5 d B)$. Similarly, when 

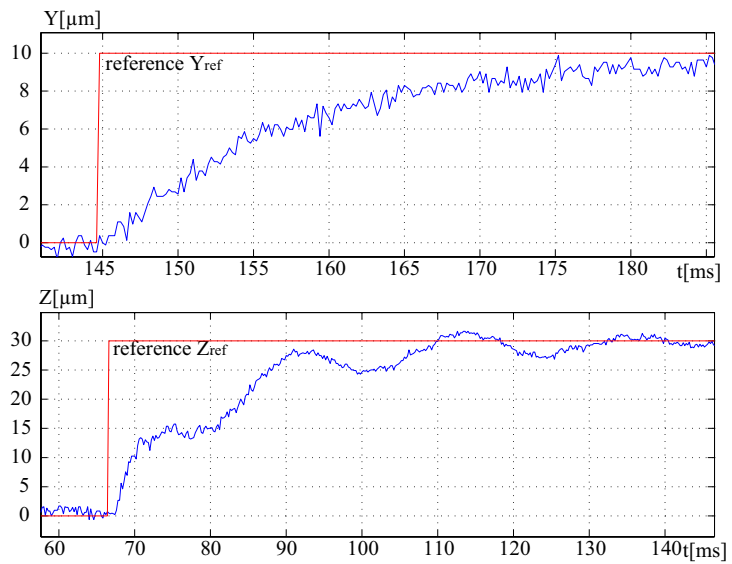

Fig. 15. Experimental steps response of the closed-loop piezocantilever: zoom

a sine input $Z_{\text {ref }}=30 \mu \mathrm{m}$ is applied, its effect on $Y$ is largely rejected (Fig. 16-b) as $20 \log \left(\frac{Y}{Z_{\text {ref }}}\right)$ is nearly $-30 d B$.
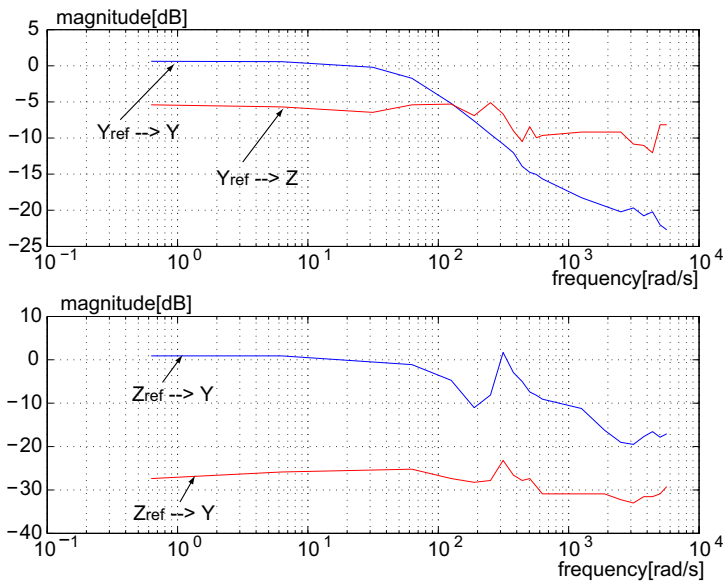

Fig. 16. Experimental harmonic analysis of the closed-loop piezocantilever.

\section{CONClusion}

The scope of this paper is the characterization, modeling and control of a 2-dof piezocantilever dedicated to micromanipulation/microassembly tasks. In addition to the hysteresis and the creep, the piezocantilever presents a coupling in the two axis that can compromise accurate tasks. Hence, a model was developed and a controller was synthesized. In order to account the hysteresis, the creep, the coupling and the performances specifications, a $H_{\infty}$ robust controller was applied. The experimental results show the efficiency of the method and indicate that the obtained performances are well suited to the micromanipulation/microassembly requirements.

\section{ACKNOWLEDGMENT}

This work is partially supported by the French research project NANOROL (ANR $N^{\circ}$ PSIROB07_184846).

\section{REFERENCES}

[1] Y. Haddab, N. Chaillet and A. Bourjault, 'A microgripper using smart piezoelectric actuators', IEEE/RSJ IROS, Takamatsu Japan, October-November 2000.

[2] D. J. Capparelli, M. I. Frecker, T. W. Simpson and A. Snyder, 'Design of a PZT bimorph actuator using a metamodel-based approach', Transactions of the ASME, Journal of Mechanical Design, pp.354-357, 2002.

[3] H. Xinhan, C. Jianhua, W. Min and L. Xiadong, 'A piezoelectric bimorph micro-gripper with micro-force sensing', IEEE ICIA, June-July 2005.

[4] J. Agnus, P. Nectoux and N. Chaillet, 'Overview of microgrippers and design of a micro-manipulation station based on a MMOC microgripper', IEEE CIRA, Espoo Finland, june 2005.

[5] M. Rakotondrabe, C. Clévy and P. Lutz, 'Modelling and robust position/force control of a piezoelectric microgripper', IEEE CASE, Scottsdale AZ USA, pp.39-44, September 2007.

[6] M. Rakotondrabe, Y. Haddab and P. Lutz, 'Modelling and $H_{\infty}$ force control of a nonlinear piezoelectric cantilever', IEEE/RSJ IROS, San Diego CA USA, pp.3131-3136, October-November 2007.

[7] S. Devasia, E. E. Eleftheriou, R. Moheimani, 'A survey of control issues in nanopositioning', IEEE Transactions on Control Systems Technology, Vol.15, $N^{o} 15$, pp.802-823, 2007.

[8] P. De Lit, J. Agnus, C. Clévy and N. Chaillet, 'A four-degreeof-freedom microprehensile microrobot on chip', pp.33-42, International Journal of Assembly Technology and Management (Assembly Automation), 2004

[9] J. Agnus, P. De Lit, C. Clévy and N. Chaillet, 'Description and performances of a four-degrees-of-freedom pizoelectrique microgripperp', IEEE ISATP, pp.66-71, France, 2003.

[10] R. Pérez, J. Agnus, C. Clévy, A. Hubert and N. Chaillet, 'Modeling, fabrication and validation of a high-performance 2dof piezactuator for micromanipulation', IEEE/ASME Transactions on Mechatronics, pp.161-171, April 2005.

[11] M. Rakotondrabe, Y. Haddab and P. Lutz, 'Quadrilateral modelling and robust control of a nonlinear piezoelectric cantilever', IEEE Transactions on Control Systems Technology, Vol.17, No3, pp.528-539, May 2009.

[12] N. C. Singer, W. P. Seering and K. A. Pasch, 'Shaping command inputs to minimize unwanted dynamics', Patent $N^{\circ}$ US4.916.635, 1990.

[13] D. O. Popa, B. H. Kang, J. T. Wen, H. E. Stephanou, G. Skidmore and A. Geisberger, 'Dynamic modeling and input shaping of thermal bimorph mems actuators', IEEE ICRA, 2003.

[14] M. Rakotondrabe, C. Clévy and P. Lutz, 'Hysteresis and vibration compensation in a nonlinear unimorph piezocantilever', IEEE/RSJ IROS, Nice France, 2008.

[15] D. Croft, G. Shed and S. Devasia, 'Creep, hysteresis and vibration compensation for piezoactuators: atomic force microscopy application', ASME Journal of Dynamic Systems, Measurement and Control, 2001.

[16] T. S. Low and W. Guo, 'Modeling of a three-layer piezoelectric bimorph beam with hysteresis', Journal of MicroElectroMechanical Systems, pp.230-237, 1995.

[17] G. J. Balas, J. C. Doyle, K. Glover, A. Packard and R. Smith, ' $\mu$-analysis and synthesis toolbox', The Matworks User's Guide3, 2001.

[18] K. Glover and J. C. Doyle, 'State-space formulae for all stabilizing controllers that satisfy an $H_{\infty}$-norm bound and relations to risk sensivity', Systems \& Control Letters, vol.11, pp.167$172,1988$.

[19] J. C. Doyle, K. Glover, P. K. Khargonekar and B. A. Francis, 'State-space solutions to standard $\mathrm{H}_{2}$ and $\mathrm{H}_{\infty}$ control problems', IEEE Transactions on Automatic Control, AC 34 Nř8, pp.831-846, 1989.

[20] B. C. Moore, 'Principal component analysis in linear systems: controllability, observability and model reduction', IEEE Transactions on Automatic Control, AC-26(1), 1981. 